\title{
Capillary blood cultures
}

\author{
R. J. HOLT, C. H. FRANKCOMBE, and R. L. NEWMAN* \\ From the Group Laboratories, Queen Mary's Hospital for Children, Carshalton, Surrey
}

\begin{abstract}
Holt, R. J., Frankcombe, C. H., and Newman, R. L. (1974). Archives of Disease in Childhood, 49, 318. Capillary blood cultures. The need for frequent blood cultures from small babies for the early detection of bacteraemia associated with internal prosthesis colonization led to the development of a method for capillary blood collection. Fingers, toes, or heels were sterilized as well as possible, the surface was coated with sterile petroleum jelly, and sufficient blood was collected aseptically for aerobic broth culture and agar pour plates.

From 242 patients, mostly babies with hydrocephalus and ventriculoatrial shunts, 740 cultures were collected; $135(19 \%)$ cultures were positive and $32(4 \cdot 3 \%)$ were judged to be contaminated. A comparison between the results of capillary and venous blood cultures is made.
\end{abstract}

Bacteraemia is often the first sign of bacterial colonization of ventriculoatrial shunts implanted in young babies to alleviate hydrocephalus, but the clinical and haematological signs of somatic response to this infection are frequently minimal. Frequent blood cultures are therefore necessary for early detection of this complication, which appears at some stage in at least $10 \%$ of all children with this internal prosthesis (Eckstein and Cooper, 1968), and which is almost always caused by coagulase-negative staphylococci already resident on the child (Holt, 1969). It follows that not only must blood cultures be taken frequently over long periods, sometimes for several years, but that they should be reliably free from contamination by resident skin bacteria. When conventional venous blood collection is used these requirements present considerable problems unless done by highly skilled and experienced staff, fully aware of the misleading results that are likely to follow casual contamination; even then aseptic venepuncture is not easy in small debilitated babies, and contamination hazards increase sharply when femoral vein puncture is used. These considerations led us 4 years ago to experiment with the collection of blood from pricks in prepared skin areas on the fingers, toes, and heels of infants with implanted shunts, and this paper reports the techniques that evolved, together with results from about 700 such cultures.

\footnotetext{
Received 1 October 1973.

*Now deceased.
}

Sir Almroth Wright devised his blood capsule for the collection of peripheral blood at the beginning of the century (Wright, 1909) and certainly in the 1930s blood cultures were still being made in some laboratories on the blood clots separated in the capsules; one of us (R.J.H.) repeatedly saw Salmonella sp. recovered by this means at that time, and it is of interest historically that the first positive culture of our series was performed on a child with Salmonella paratyphi B septicaemia.

\section{Method}

Procedures 1-6 were followed throughout this series of investigations.

(1) The outer fleshy curve of the big toe, occasionally the thumb, or in very small babies the heel, was thoroughly cleaned first with sterile saline and then dried with sterile gauze; it was then cleaned with a Medi-Swab (a tissue soaked in isopropyl alcohol) and allowed to dry. At this stage a pad culture (Holt, 1966) could be made of the surface to see whether any bacteria survived, followed by a second pad culture after collection was complete.

(2) A thin, even layer of sterile petroleum jelly was applied to the sterilized skin surface with a sterile spatula. The petroleum jelly was distributed in $1 \mathrm{ml}$ quantities into $6 \mathrm{ml}$ 'bijou' bottles, and sterilized by dry heat.

(3) A vertical prick was made with one of the medical lancets in general use.

(4) Providing that the toe was held steady with the sterilized surface upwards, small beads of blood could collect on the surface of petroleum jelly with a minimum of pressure to the rest of the toe. This blood was collected by capillary flow into a sterile pipette, 
TABLE I

Capillary blood cultures taken between 1970 and 1973

\begin{tabular}{|c|c|c|c|c|c|}
\hline \multirow{2}{*}{ Cases } & \multirow{2}{*}{$\begin{array}{l}\text { No. of } \\
\text { cultures }\end{array}$} & \multirow{2}{*}{$\begin{array}{l}\text { No. of } \\
\text { patients }\end{array}$} & \multicolumn{2}{|c|}{ Positive results } & \multirow{2}{*}{$\begin{array}{l}\text { Cultures considered } \\
\text { contaminated (and \%) }\end{array}$} \\
\hline & & & $\begin{array}{l}\text { Liquid culture } \\
\text { (and } \% \text { ) }\end{array}$ & $\begin{array}{l}\text { Pour plate culture } \\
\text { (and \%) }\end{array}$ & \\
\hline $\begin{array}{l}\text { Ventriculoatrial shunt } \\
\text { Others (leukaemia, suspected } \\
\text { Gram-negative septicaemia, } \\
\text { candidaemia, etc.) }\end{array}$ & $\begin{array}{l}635 \\
105\end{array}$ & $\begin{array}{r}201 \\
41\end{array}$ & $\begin{array}{c}113 \\
(17 \cdot 8) \\
22 \\
(21)\end{array}$ & $\begin{array}{c}74 \\
(11 \cdot 6) \\
15 \\
(14 \cdot 2)\end{array}$ & $\begin{array}{c}29 \\
(4 \cdot 6) \\
3 \\
(2 \cdot 8)\end{array}$ \\
\hline
\end{tabular}

preferably graduated so that some indication of quantity could be obtained. When 0.3 to $0.5 \mathrm{ml}$ blood had been collected, this was expelled into about $3 \mathrm{ml}$ of sterile infusion broth, which was taken immediately to the laboratory.

(5) $0.5 \mathrm{ml}$ of this blood suspension was incorporated into $20 \mathrm{ml}$ infusion broth agar and a pour plate was made. After 48 hours aerobic incubation, all visible colonies were counted and calculation of viable bacterial aggregates per $\mathrm{ml}$ in the original blood was done.

(6) $2.5 \mathrm{ml}$ of the blood suspension was added to about $50 \mathrm{ml}$ infusion broth; penicillinase or PABA was added as indicated, though we believe that the dilution is sufficient to reduce any antibacterial substances to below inhibitory levels. The blood cultures were incubated aerobically for 10 days and subcultured onto blood agar after 1, 2, 3, and 10 days incubation.

For some purposes a greater proportion of the blood suspension could be incorporated in the pour plate; in the present series the pour plate culture was used principally to give early warning of very heavy bacteraemia, and broth cultures were regarded as the basic diagnostic method. Antibiotic sensitivity discs containing cloxacillin or gentamicin were occasionally placed on the pour plates in the hope that rapid guidance for suitable therapy might be obtained.

These manipulations present no problem to an experienced technician; the major difficulty is in ensuring that the small drops of blood do not flow away from the incision site, because if they leave the coated surface the risk of contamination with cutaneous flora will increase sharply. Other grease formulations have been tried, including some containing silicone derivatives, but they had no advantage over sterile petroleum jelly for establishing a sterile water repellent surface on which beads of blood could sit.

\section{Results}

Almost all patients were under 2 years of age and most were less than 6 months. Only occasionally was 1 culture taken; most had 2 cultures taken, though occasionally 3 or 4 or more, up to 10 , were collected.

Capillary blood cultures, 1970-73. The results of 740 cultures taken during this period are given in Table I. Originally the intention was to compare at least the first 100 capillary cultures with nearly simultaneous venepunctures, but this proved to be impractical because not every capillary culture was shortly followed or preceded by a venepuncture culture. On the rare occasions when it was possible to take pairs of cultures close together a close correlation resulted.

Nevertheless, it is important to compare, if possible, the contaminant risk met in the two methods, and the results for one year's venepuncture cultures are presented in Table II.

TABLE II

Venepuncture blood cultures, 1972

\begin{tabular}{l|c|c|c}
\hline \multicolumn{1}{c|}{ Cases } & $\begin{array}{c}\text { No. of } \\
\text { cultures }\end{array}$ & $\begin{array}{c}\text { No. of } \\
\text { patients }\end{array}$ & $\begin{array}{c}\text { Cultures considered } \\
\text { contaminated } \\
\text { (and \%) }\end{array}$ \\
\hline With shunts & 290 & 130 & $\begin{array}{c}22 \\
7 \cdot 5) \\
49\end{array}$ \\
Others & 1255 & 571 & $(3 \cdot 9)$ \\
\hline
\end{tabular}

Replicate pad cultures of skin surface before and after capillary collection. The first 184 collections from 80 children were examined in this way. 4 positive pad cultures were found in the series taken before puncture, revealing mixed cultures of resident skin bacteria; 6 taken after blood collection were positive, 3 yielding growth of the biotype of staphylococcus recovered from the blood culture. The smear of petroleum jelly on the pads taken after blood collection was transferred to the replicate culture plates but did not appear to make plate reading difficult.

Pour plate cultures. It was not always possible to collect sufficient capillary blood for culture in both broth and pour plates; therefore, of 
740 cultures taken, only 525 were cultured in both. These results are shown in Table III.

\section{TABLE III}

Results of capillary blood cultures in broth and pour plates

\begin{tabular}{|c|c|c|c|c|}
\hline \multicolumn{2}{|c|}{$\begin{array}{l}\text { Cultured in broth } \\
\text { only }\end{array}$} & \multicolumn{3}{|c|}{ Cultured in broth and pour plates } \\
\hline Total & Positive & Total & $\begin{array}{l}\text { Positive broth, } \\
\text { positive plate }\end{array}$ & $\begin{array}{l}\text { Positive broth, } \\
\text { negative plate }\end{array}$ \\
\hline 215 & 31 & 525 & 89 & 15 \\
\hline
\end{tabular}

Pour plate counts. A wide range of counts $/ \mathrm{ml}$ viable bacterial units was noted in the 89 positive pour plate cultures. The highest was calculated at 2800 colonies $/ \mathrm{ml}$. There was no means of determining whether each colony originated from one bacterium or from a single viable aggregate.

Species recovered. Staphylococcus albus was by far the most common organism in our series, reflecting the type of patient for whom the technique was initially devised, but on several occasions Staphylococcus aureus, Pseudomonas aeruginosa, and various Enterobacteriaceae were grown in broth and pour plate. On a few occasions Streptococcus viridans was recovered, and once Haemophilus influenzae, in each case appearing only from the broth. Candida albicans was grown in 3 broth cultures, and once in a pour plate in considerable numbers, revealing a heavy candidaemia.

\section{Discussion}

The practical advantages to the clinician of a capillary blood culture method are evident: repeated cultures can be taken at short intervals from the fingers, toes, and heels of even very small babies by judicious alternation. In much the same way we have for many years collected sufficient blood for serum electrolyte estimation whenever we felt unable to perform further venepunctures on small and very sick infants, though in these investigations sterile collection is not so essential. The experience of our technical staff in the latter procedures has greatly helped their approach to sterile blood collection, though it still requires considerable skill and individual ability and not all staff proved able to make reliably sterile collections from small and difficult patients.

Far more significant than the ease of collection is the reliability of the results of the cultures, and there are two questionable aspects of these results. First, it is important to ascertain that culture results from capillary blood collected from extremities agree with those taken from veins, and that bacteria present in central venous blood are capable of penetrating into remote peripheral sites. Presumably they can, for they are rarely larger than red blood cells, but it is conceivable that many retention mechanisms may hinder their passage into tissue. In this series the vast majority of our capillary cultures have yielded the same bacterial biotypes found in the patient's blood before or after the peripheral collection. Nevertheless, to assert that blood cultures from both sites are always simultaneously positive or that venepuncture necessarily yields more positive results than capillary puncture may be overstating the case.

The second question that has confused the interpretation of blood culture results for many years is the difficulty of distinguishing between truly haematogenous micro-organisms and contaminants, either those from the subject's skin or those introduced during subsequent technical manipulations. Until recently little attention was paid by many laboratories even to heavy and repeated growths of coagulase-negative staphylococci, aerobic diphtheroids, or candida, which were dismissed as casual skin contaminants. It is now recognized that pre-existent cardiac damage or malformation and the presence of many kinds of internal artificial devices predispose greatly towards persistent bacteraemia caused by these organisms, the exact biotypes of which are frequently present on the subject's skin or nares, or in the faeces (Holt, 1969). Evaluation is further obscured because even heavy bacteraemias of this nature often evoke little somatic response and may be quite difficult to detect clinically.

The results of each blood culture have always been very carefully assessed in relation to the available clinical evidence and to other blood culture results from the same patient, and though considerable thought is given to each result, the clinical reality of the isolated positive culture remains uncertain where this is due to organisms commonly found on the skin or in the respiratory or gastrointestinal tract. These may in fact be blood borne, if only transiently, even on those rare occasions when two different bacterial species are isolated concurrently. Though capillary cultures appeared to be less contaminated $(4 \cdot 6 \%)$ than cultures from venepunctures $(7 \cdot 5 \%)$, this was probably caused by the fact that many of the latter collections were from extremely difficult cases, and were often by femoral vein puncture in small, gravely ill babies.

The value of the pour plate was in far less doubt; 
whenever several colonies of a single bacterial species appeared in different parts of the plate this was thought to present strong evidence of true and usually heavy bacteraemia. High colony counts were almost always accompanied by repeatedly positive venous blood cultures with the same organism, which was nearly always eliminated from the blood by chemotherapy.

In a further series, capillary blood samples may be further divided into anaerobic cultures in addition to those detailed here, and thus permit comparison with the routine aerobic and anaerobic cultures on venous blood. The purposes of this study were, however, to look for the aerobic micro-organisms implicated in shunt colonization and to test the feasibility of the concept of capillary blood cultures on a routine scale.

Most of these investigations were carried out in the lifetime of Dr. R. L. Newman, to whom a generous grant was made by the Spina Bifida Trust for research into the problems of colonization of ventricular shunt systems; much of it was used to finance necessary technical assistance. We are grateful for the co-operation of $\mathrm{Mr}$. H. B. Eckstein and Mr. D. M. Forrest, paediatric surgeons to this hospital, and of their clinical and nursing teams.

\section{REFERENCES}

Eckstein, H. B., and Cooper, D. G. W. (1968). The complications of ventriculo-atrial shunts with the Holter valve for hydrocephalus. Zeitschrift für Kinderchirurgie und Grenzgebiete, 5, 309.

Holt, R. J. (1966). Pad culture studies on skin surfaces. Fournal of Applied Bacteriology, 29, 625.

Holt, R. (1969). The classification of staphylococci from colonized ventriculo-atrial shunts. Fournal of Clinical Pathology, 22, 475.

Wright, A. E. (1909). Studies on Immunization. Constable, London.

Correspondence to Dr. R. J. Holt, Group Laboratories, Queen Mary's Hospital for Children, Carshalton, Surrey. 